Revue d'histoire de l'Amérique française

REVUE D.HISTOIRE DE L'AMÉRIQUE FRANÇAISE

\title{
Vendre la banlieue aux Montréalais : discours et stratégies publicitaires, 1950-1970
}

\section{Harold Bérubé}

Volume 71, numéro 1-2, été-automne 2017

URI : https://id.erudit.org/iderudit/1042788ar

DOI : https://doi.org/10.7202/1042788ar

Aller au sommaire du numéro

\section{Éditeur(s)}

Institut d'histoire de l'Amérique française

ISSN

0035-2357 (imprimé)

1492-1383 (numérique)

Découvrir la revue

Citer cet article

Bérubé, H. (2017). Vendre la banlieue aux Montréalais : discours et stratégies publicitaires, 1950-1970. Revue d'histoire de l'Amérique française, 71(1-2),

83-112. https://doi.org/10.7202/1042788ar
Résumé de l'article

Cet article traite de la façon dont est mise en marché la banlieue de masse à Montréal dans les années 1950 et 1960. En analysant les publicités relatives aux résidences unifamiliales neuves dans les journaux francophones et anglophone de la métropole, je cherche à mieux comprendre comment évoluent les stratégies publicitaires mises de l'avant et comment se structure l'idéal suburbain qui s'en dégage. L'analyse permet de faire ressortir ce qui constitue une publicité idéaltypique pour la période étudiée, mais également de constater que le discours et les stratégies utilisés se complexifient au fil des ans et convergent d'un groupe linguistique à l'autre, du moins pour ce qui est de leur contenu. 


\title{
Vendre la banlieue aux
}

Montréalais : discours et stratégies publicitaires, I950-1970

\author{
HaRold BÉRUbÉ \\ Université de Sherbrooke
}

RÉsumÉ - Cet article traite de la façon dont est mise en marché la banlieue de masse à Montréal dans les années 1950 et 1960. En analysant les publicités relatives aux résidences unifamiliales neuves dans les journaux francophones et anglophone de la métropole, je cherche à mieux comprendre comment évoluent les stratégies publicitaires mises de l'avant et comment se structure l'idéal suburbain qui s'en dégage. L'analyse permet de faire ressortir ce qui constitue une publicité idéaltypique pour la période étudiée, mais également de constater que le discours et les stratégies utilisés se complexifient au fil des ans et convergent d'un groupe linguistique à l'autre, du moins pour ce qui est de leur contenu.

ABSTRACT - This article addresses the way mass suburbanization was marketed to Montrealers in the 1950s and 1960s. By analyzing advertisements for newly-built single-family homes in Anglophone and Francophone newspapers, I seek to better understand the evolution of marketing strategies and the structuring of their underlying suburban ideal. The analysis allows me not only to shed light on what constitutes the ideal-typical publicity for this period, but also to observe how the discourses and strategies were complexified over the years and converged between both linguistic groups, at least in their content.

1. Je tiens d'abord à remercier Dominique Foisy-Geoffroy, qui m'a lancé sur cette piste en 2013. J'aimerais aussi remercier les assistants de recherche qui m’ont aidé à rassembler les données utilisées ici: Benoît Marsan, Alexandre Patenaude, Jérémie Rose et Alexandre Leclerc. Je remercie également le Laboratoire d'histoire et de patrimoine de Montréal qui m'a soutenu dans ce projet, et en particulier Kim Petit et Laura Barreto qui ont joué un rôle central dans la géolocalisation des données. J’aimerais enfin remercier Amélie Bourbeau, qui a commenté une première version de ce texte, ainsi que les évaluateurs anonymes de la revue pour leurs commentaires constructifs. 


\section{INTRODUCTION}

Dans l'introduction de son recueil d'essais Recours aux sources, l'historien Éric Bédard revient sur l'importance accordée, dans la mémoire populaire, à l'année 1960 comme date d'entrée du Québec dans la modernité. Il explique notamment que, pour «de jeunes parents du milieu ouvrier qui achetaient leur premier bungalow alors que, dans les années 1930, ils avaient grandi dans les taudis de Saint-Henri, le Québec des années 1950 était rempli de promesses $^{2}$ ». Difficile de nier, effectivement, que pour la grande majorité des Nord-Américains issus des couches populaires, l'accès à la propriété dans les années d'après-guerre fut considéré comme un important symbole d'accession à la classe moyenne. Dans un texte publié en 2014, l'historien Michel Lessard va dans le même sens lorsqu'il décrit avec nostalgie les après-midi passés par ses parents et lui, en 1949-1950, à visiter des maisons modèles à la recherche de la demeure qui leur permettra d'échapper à leur étroit logement du temps de la guerre: «[mes] parents, ma mère surtout rêvait d’un de ces bungalows modernes, équipés d'une cuisine fonctionnelle, d'une salle de bain en céramique, de chambres à coucher dotées de garde-robes pour chacun de ses trois enfants, d'un beau grand salon pour recevoir la visite, et d'une salle de télévision, une nouveauté sur le point de conquérir tous les foyers». Il ajoute que ce rêve, «[journaux] et magazines en faisaient largement une promotion attrayante $»^{3}$.

C'est à cette "promotion attrayante " que je m'intéresse d'abord et avant tout dans cet article. On a beaucoup disserté, depuis les années 1990, sur le rapport de la société québécoise à l’américanité et à la modernité ${ }^{4}$. À leur façon, la suburbanisation, comme réalité matérielle de masse, et l'idéal suburbain, comme aspiration diffusée largement dans les médias, représentent d'importants marqueurs de l'inscription de la société québécoise dans une certaine modernité nord-américaine d’après-guerre. Plus spécifiquement - car le terme «modernité » demeure aussi utile que flou ${ }^{5}-$, on peut

2. Éric Bédard, Recours aux sources. Essais sur notre rapport au passé (Montréal, Boréal, 2011), p. 13.

3. Michel Lessard, «La meilleure maison des jeunes familles d'aujourd'hui: les bungalows», Le Peuple Lévis, 11 juin 2014, p. 6. Je remercie Alex Tremblay de m'avoir signalé l'existence de ce texte.

4. Il y aurait des dizaines d'études auxquelles renvoyer le lecteur. Je me contenterai d'évoquer certains des ouvrages les plus importants sur ces questions: Gérard Bouchard et Yvan Lamonde, dir., Québécois et Américains. La culture québécoise aux XIXe et XXe siècles (Montréal, Fides, 1995); Joseph Yvon Thériault, Critique de l'américanité. Mémoire et démocratie au Québec (Montréal, Québec Amérique, 2002); Yvan Lamonde, La modernité au Québec. Tome 1. La Crise de l'homme et de l'esprit (1929-1939) (Montréal, Fides, 2011) et Yvan Lamonde, La modernité au Québec. Tome 2. La victoire différée du présent sur le passé (1939-1965) (Montréal, Fides, 2016).

5. Jean-Philippe Warren, "Petite typologie philologique du "moderne" au Québec (1850-1950). Moderne, modernisation, modernisme, modernité», Recherches sociographiques, 46, 3 (septembredécembre 2005), p. 495-525. 
parler de l'adhésion de la société québécoise à cette république des consommateurs qu'a décrite et analysée Lizabeth Cohen ${ }^{6}$. Si cette adhésion ne me semble pas faire de doute, ses modalités demeurent peu étudiées et c'est une réflexion à laquelle j'aimerais contribuer dans les pages qui viennent. En m'intéressant à la façon dont est mise en marché la banlieue de masse d'après-guerre à Montréal, je veux comprendre comment se structure l'idéal suburbain dans ce contexte. Plus précisément, il s'agira d'analyser ce discours publicitaire entre 1950 et 1970 en étant particulièrement attentif à la façon dont il évolue dans le temps de façon générale, mais aussi selon qu’il s'adresse à l'un ou l'autre des deux grands groupes linguistiques de la métropole montréalaise.

Je commencerai donc par situer ma réflexion sur le plan historiographique et méthodologique. On verra ensuite que l'analyse permet de faire ressortir ce qui constitue en quelque sorte une publicité idéaltypique pour la période étudiée, publicité dont les principales caractéristiques varient assez peu durant les deux décennies couvertes. Je compléterai cette analyse par une réflexion sur les éléments qui, malgré cette toile de fond stable, changent dans le temps et selon le groupe linguistique auquel s'adressent les publicitaires. On verra, globalement, que le discours et les stratégies utilisés se complexifient au fil des ans et convergent, du moins pour ce qui est de leur contenu, dans les quotidiens anglophone et francophones de la métropole.

\section{BANLIEUES ET PUBLICITÉS, MODERNITÉ ET AMÉRICANITÉ}

Historiographiquement, cet article se situe au carrefour de l'histoire de la consommation et de l'histoire urbaine. Dans le premier cas, on parle d'un champ de recherche encore relativement jeune au Québec ${ }^{7}$. Dans un numéro de la Revue d'histoire de l'Amérique française consacré à la question, Magda Fahrni définit largement la consommation comme le fait de "participer au marché et aux transactions commerciales», et souligne l'importance pour les historiens qui l'étudient de s'intéresser à son sens,

6. Il faudrait évidemment nuancer: Cohen évoque les liens étroits qui sont tissés durant cette période entre la consommation et les valeurs républicaines de liberté et d'égalité, une réalité qui ne se présente pas de la même façon au Canada et au Québec. Il y aurait certainement lieu d'explorer plus avant l'idée d'un «Consumers' Dominion». Lizabeth Cohen, A Consumers' Republic. The Politics of Mass Consumption in Postwar America (New York, Random House, 2003).

7. Soulignons tout de même l'importante thèse que consacre à la question Stéphanie O'Neill, "L'argent ne fait pas le bonheur: les discours sur la société de consommation et les modes de vie à Montréal, 1945-1975 », thèse de doctorat (histoire), Université de Montréal, 2016. 
notamment comme "stratégie de distinction sociale »" Il est clair, dans ce contexte, que l'achat d'une résidence constitue, pour la majorité des consommateurs québécois de l'après-guerre, la plus importante participation au marché de leur vie sur le plan financier. Ajoutons que l'achat d'une telle demeure va de pair avec l'acquisition d'un nombre de biens et d'accessoires qui ira en augmentant au fil des Trente Glorieuses?.

L'étude de la publicité et des stratégies de vente s'inscrit nettement dans cette histoire de la consommation. Différentes monographies générales ont été consacrées à la question aux États-Unis et au Canada ${ }^{10}$. Au Québec, mentionnons la récente contribution de Sébastien Couvrette qui lie intimement l'évolution générale du discours publicitaire dans les grands quotidiens montréalais entre 1920 et 1970 à la formation de la classe moyenne ${ }^{11}$. Toutefois, il est assez peu disert pour ce qui est spécifiquement de la question de la propriété immobilière. Dans une courte section, il souligne que, avant la Seconde Guerre mondiale, la propriété apparaît comme un «symbole du statut de la classe moyenne aisée» et que les publicités des années 1930 relatives aux banlieues témoignent d'un idéal suburbain tout de même modeste $^{12}$. Cette modestie est toujours palpable dans les années $1950^{13}$, mais on verra qu'elle s'atténue considérablement par la suite.

Cela dit, c'est d'abord et avant tout en tant qu'historien urbain - ou suburbain - que j'aborde cet objet de recherche. À cet égard, force est d'avouer que plusieurs facettes de l'histoire des banlieues d'après-guerre,

8. Magda Fahrni, «Explorer la consommation dans une perspective historique », Revue d'histoire de l'Amérique française, 58, 4 (printemps 2005), p. 466.

9. Sur cette question, se référer aux travaux de Daniel Miller, et notamment à The Comfort of Things (Cambridge, Polity, 2009). Cela dit, O’Neill relativise, avec raison, l'importance de ces indicateurs de l'entrée dans la société de consommation dans le cas québécois. S. O’Neill, «L'argent ne fait pas le bonheur...", p. 90-98.

10. Voir notamment, dans le cas canadien, Luc Côté et Jean-Guy Daigle, Publicité de masse et masse publicitaire: le marché québécois des années 1920 aux années 1960 (Ottawa, Presses de l'Université d'Ottawa, 1999) et Russell Johnston, Selling Themselves : The Emergence of Canadian Advertising (Toronto, University of Toronto Press, 2001); et, dans le cas américain, Roland Marchand, Advertising the American Dream: Making Way for Modernity, 1920-1940 (Berkeley, University of California Press, 1985), Stuart Ewen, Captains of Consciousness: Advertising and the Social Roots of the Consumer Culture (New York/Toronto, McGraw-Hill, 1976) et Stephen Fox, The Mirror Makers. A History of American Advertising and Its Creators (Urbana, University of Illinois Press, 1997).

11. Sébastien Couvrette, Le récit de la classe moyenne. La publicité des quotidiens montréalais, 1920-1970 (Montréal, Leméac, 2014).

12. S. Couvrette, Le récit de la classe moyenne..., p. 186.

13. «Pour un grand nombre de familles, l'augmentation du niveau de vie dans les années d'aprèsguerre permet simplement de subvenir à leurs besoins fondamentaux, non pas de participer activement à la société de consommation et des loisirs, comme le suggère la publicité», S. Couvrette, Le récit de la classe moyenne..., p. 186. Voir également Valerie Korinek, Roughing it in the Suburbs. Reading Chatelaine Magazine in the Fifties and Sixties (Toronto, University of Toronto Press, 2000). 
au Québec comme au Canada, mériteraient plus d'attention. D’une certaine façon, l'excellente synthèse produite sur le sujet par Richard Harris en 2004 donne le ton. L'ouvrage, qui couvre les 60 premières années du $\mathrm{XX}^{\mathrm{e}}$ siècle, se penche longuement sur les racines complexes du processus de suburbanisation au Canada, mettant en relief la diversité initiale du paysage suburbain et la relative précarité de plusieurs de ses premiers promoteurs ${ }^{14}$. Harris conclut sa démonstration avec les années 1950, assumant qu'une fois que le processus s'est massifié et standardisé, il n'y a plus grand-chose d'intéressant à dire à son sujet ${ }^{15}$. L'historiographie proprement montréalaise semble lui faire écho. Des travaux pionniers de Paul-André Linteau sur Maisonneuve à mes propres recherches sur les banlieues bourgeoises, en passant par l'ouvrage de Robert Lewis sur les banlieues industrielles et ouvrières de la métropole, ce sont clairement les années d'avant-guerre qui ont été privilégiées jusqu'ici par une partie importante des chercheurs ${ }^{16}$. C'est doublement vrai si on s'intéresse plus spécifiquement à l'histoire culturelle de cette suburbanisation d'après-guerre.

Les meilleures études canadiennes et québécoises sur l’histoire sociale et culturelle de ces banlieues des Trente Glorieuses sont probablement celles qui traitent de la place qu'y occupent les femmes, notamment en écho à des essais comme celui de Betty Friedan, The Feminine Mystique ${ }^{17}$. Mais pour trouver des travaux similaires à ceux produits aux États-Unis sur les représentations plus générales de la banlieue d'après-guerre ${ }^{18}$, il

14. Richard Harris, Creeping Conformity. How Canada Became Suburban, 1900-1960 (Toronto, University of Toronto Press, 2004).

15. Voir la critique de l'ouvrage proposée par le géographe Larry McCann, «A Regional Perspective on Canadian Suburbanization: Reflections on Richard Harris's Creeping Conformity", Urban History Review/Revue d'histoire urbaine, 35, 1 (2006), p. 32-45.

16. Paul-André Linteau, Maisonneuve ou comment des promoteurs fabriquent une ville, 1883-1918 (Montréal, Boréal, 1981); Harold Bérubé, Des sociétés distinctes. Gouverner les banlieues bourgeoises de Montréal, 18801939 (Montréal et Kingston, McGill-Queen’s University Press, 2014); Robert Lewis, Manufacturing Montreal. The Making of an Industrial Landscape, 1850 to 1930 (Baltimore, Johns Hopkins University Press, 2000). Il faut néanmoins souligner qu'il y a d'importantes exceptions, comme Jean-Pierre Collin, La cité coopérative canadienne-française. Saint-Léonard-de-Port-Maurice, 1955-1963 (Montréal, Presses de l'Université du Québec, 1986).

17. Le célèbre essai de Friedan dénonçait le «renfermement» des femmes dans les banlieues nordaméricaines et de nombreuses études ont fait écho à son analyse, ou alors ont nuancé ses conclusions sur le sujet. Betty Friedan, The Feminine Mystique (New York, W. W. Norton \& Company, 2001 [1963]). Ces études sont nombreuses et je me contente d'évoquer certaines des principales contributions à ces recherches: Anne-Marie Séguin, "Madame Ford et l'espace: lecture féministe de la suburbanisation", Lieux et milieux de vie, 2, 1 (1989), p. 51-68; Veronica Strong-Boag, "Home Dreams: Women and the Suburban Experiment in Canada, 1945-1960", Canadian Historical Review, 72, 4 (décembre 1991), p. 471504 ; V. Korinek, Roughing it in the Suburbs....

18. Voir notamment Dolores Hayden, “I HAVE SEEN THE FUTURE” : Selling the Unsustainable City ", Journal of Urban History, 38, 1 (2012), p. 3-15; John Archer, Architecture and Suburbia. From English Villa to American Dream House, 1690-2000 (Minneapolis, University of Minnesota Press, 2005); Becky M. 
faut sortir de la discipline historique. Je pense aux travaux menés par le Groupe interdisciplinaire de recherche sur les banlieues de l'Université Laval, qui s'est penché sur le vieillissement et la transformation de banlieues pavillonnaires de la Vieille Capitale datant des années 1960 et $1970^{19}$. Je pense également aux réflexions récentes proposées sur le sujet par des chercheurs en études littéraires qui constatent notamment l'émergence tardive, mais indéniable, de la banlieue comme décor pour un nombre grandissant de romans québécois ${ }^{20}$. Néanmoins, pour trouver une étude à laquelle mes recherches font véritablement écho, il faut remonter un peu plus loin dans le passé.

En 1982, le sociologue québécois Gérald Fortin a produit un rapport de recherche fouillé sur les publicités relatives au logement neuf dans la région de $\mathrm{Montréal}^{21}$. Si ses recherches n'avaient pas, à proprement parler, une dimension historique, elles traitent assez largement des années 1970 et s'aventurent prudemment du côté des deux décennies précédentes. Fortin y souligne notamment l'importante valeur, sur le plan identitaire, de la résidence et le rôle central que joue la publicité dans la constitution de ce capital symbolique ${ }^{22}$. Dans ce contexte, il distingue publicité sur l'habiter et publicité sur l'habitation, la première faisant référence à la maison en tant que telle, alors que la seconde traite des biens relatifs à l'occupation de la résidence. Fortin souligne d'ailleurs que, dans ce second cas, l'espace résidentiel est étroitement associé aux femmes ${ }^{23}$. En étudiant ces publicités, Fortin voulait suivre l'évolution des valeurs associées au logement neuf dans la région métropolitaine. Ses travaux lui permettent de constater, notamment, l'important enracinement de l'idéal suburbain à la fin des années $1970^{24}$. Les recherches de Fortin ont toutefois d'impor-

Nicolaides, «How Hell Moved from the City to the Suburbs. Urban Scholars and Changing Perceptions of Authentic Community", dans Kevin M. Kruse et Thomas J. Sugrue, dir., The New Suburban History (Chicago, University of Chicago Press, 2006), p. 80-98.

19. Andrée Fortin, Carole Després et Geneviève Vachon, dir., La banlieue revisitée (Québec, Éditions Nota Bene, 2002).

20. Voir Bertrand Gervais, Alice van der Kei et Marie Parent, dir., Suburbia. L'Amérique des banlieues (Montréal, Figura, 2015); mais surtout Daniel Laforest, L'âge de plastique. Lire la ville contemporaine au Québec (Montréal, Presses de l’Université de Montréal, 2016). Voir également, pour ce qui est du cinéma québécois, Andrée Fortin, Imaginaire de l'espace dans le cinéma québécois (Québec, Les Presses de l’Université Laval, 2015).

21. Gérald Fortin, La publicité sur le logement neuf(Montréal, Institut national de la recherche scientifique, 1982).

22. G. Fortin, La publicité sur le logement neuf..., p. 17.

23. G. Fortin, La publicité sur le logement neuf..., p. 20.

24. Parlant d'individus interrogés en 1978, il explique que "pour la plupart d'entre eux le modèle d'habitation est d'être propriétaire d'une maison unifamiliale en banlieue», G. Fortin, La publicité sur le logement neuf..., p. 39. 
Tableau I

\section{Nombre de publicités rassemblées et analysées dans la presse anglophone et francophone de Montréal, I 95 I - 1969}

\begin{tabular}{|c|c|c|}
\hline Année & $\begin{array}{c}\text { Nombre de publicités } \\
\text { anglophones }\end{array}$ & $\begin{array}{c}\text { Nombre de publicités } \\
\text { francophones }\end{array}$ \\
\hline 1951 & 2 & 2 \\
\hline 1955 & 7 & 15 \\
\hline 1959 & 31 & 60 \\
\hline 1965 & 518 & 1182 \\
\hline 1969 & 338 & 920 \\
\hline
\end{tabular}

tantes limites. Même si son étude couvre la période 1956-1976, les années 1970 sont surreprésentées dans son corpus et il sous-estime considérablement la sophistication des publicités produites à partir de la fin des années 1950. Il ignore également celles qui sont publiées dans la presse anglophone ${ }^{25}$.

Mon propre corpus permet de combler ces lacunes et d'approfondir l'analyse. Comme Fortin, je me suis concentré sur les publicités publiées dans les grands quotidiens montréalais, mais j'ai rassemblé un échantillon plus important et j'ai intégré la presse anglophone à l'analyse (tableau 1). Le corpus utilisé est donc constitué de l'ensemble des publicités relatives à des maisons unifamiliales détachées et neuves publiées le samedi dans les pages de La Patrie (pour les années 1951 et 1955), de La Presse (pour les années 1959, 1965 et 1969) et de The Gazette (pour les années 1951, 1955, $1959,1965$ et 1969$)^{26}$.

On observe aisément l'augmentation du nombre de publicités publiées au cours de la période étudiée (qui serait encore plus importante si on comptait les publicités reprises à l'identique). Comme le souligne Couvrette,

25. G. Fortin, La publicité sur le logement neuf..., p. 59, 69.

26. Ajoutons trois précisions relatives à ce corpus. D’abord, le choix de dépouiller La Patrie pour les années 1950 et La Presse pour les années 1960 s'explique par le fait que le dépouillement s'est fait en deux temps, le projet ne devant traiter, initialement, que des années 1950. La décision d'ajouter les années 1960 à l'analyse m'a amené, dans le contexte du déclin de La Patrie durant ces années, à favoriser un grand quotidien montréalais francophone comparable, La Presse. Ensuite, le choix d'exclure les maisons semidétachées et les immeubles à appartements de l'analyse s'explique par la lourdeur déjà grande du corpus rassemblé. Un échantillon de ces publicités a tout de même été rassemblé. Enfin, les années 1960 (pour la presse francophone) et 1961 (pour la presse anglophone) ont également été dépouillées. Comme il s'agit de deux années différentes, ces publicités ne sont pas incluses dans le tableau, mais seront utilisées au fil de l'analyse. Ajoutons enfin que les publicités répétées à l'identique, qui sont de plus en plus nombreuses au fil de la période, n’ont également pas été comptabilisées. 
l'offre publicitaire varie selon les quotidiens et les périodes ${ }^{27}$ et, comme l'indique Fortin dans ses propres travaux, la grande majorité des publicités relatives au logement neuf sont publiées la fin de semaine ${ }^{28}$. De manière générale, les grands quotidiens montréalais vont concentrer ces publicités dans des sections précises du journal consacrées plus largement aux questions relatives à l'habitation, ce qui permet parfois d'intéressants croisements entre reportages et publicités. Ajoutons que je n'ai pas fait de recherches spécifiquement sur les modalités de production de ces publicités, mais Fortin explique que d'après «le responsable des cahiers publicitaire [sic] du journal La Presse, seuls quelques grands promoteurs utilisent à l'occasion les services d'une firme de publicité. Le plus souvent, l'annonceur apporte ses thèmes et la maquette est réalisée dans les ateliers du journal ${ }^{29}$.» C'est ce que suggère notamment une "publicité sur la publicité» publiée dans les pages de The Gazette en 1959 (figure 1 : à consulter en ligne) ${ }^{30}$.

\section{UN ENVIRONNEMENT PUBLICITAIRE SUBURBAIN}

Un simple survol des journaux dépouillés permet de faire ressortir une première réalité importante: l'omniprésence croissante du mode de vie suburbain dans ces grands quotidiens montréalais et leur contenu publicitaire. En effet, ces journaux consacrent de plus en plus d'espace, au fil des décennies 1950 et 1960, à l'habitation et à la vie en banlieue ${ }^{31}$. Ce faisant, ils contribuent à la propagation et à la normalisation de ce mode de vie. On le remarque dans des chroniques hebdomadaires comme celle que propose The Gazette aux «Home Builders» tout au long des années 1950. Chaque semaine, un plan est proposé pour la construction d'une maison unifamiliale détachée et est l'objet d'un commentaire du chroniqueur $^{32}$. Sur la même page, on retrouvera généralement toute une série d'articles et de publicités destinés à celui qui veut construire, rénover ou décorer sa propre maison. Les journaux font également de plus en plus

27. S. Couvrette, Le récit de la classe moyenne..., p. 34.

28. G. Fortin, La publicité sur le logement neuf..., p. 21.

29. G. Fortin, La publicité sur le logement neuf..., p. 21. Voir également Jean de Bonville, La presse québécoise de 1884 à 1914. Genèse d'un média de masse (Sainte-Foy, Les Presses de l’Université Laval, 1988), p. 324.

30. Pour des raisons d'espace et de lisibilité, il n'a pas été possible d'intégrer dans cet article toutes les figures liées à la démonstration. On trouvera ces figures manquantes à <https: / haroldberube.com/ vendre-la-banlieue-aux-montrealais $>$.

31. Soulignons d'ailleurs que si les publicités relatives à des maisons neuves sont peu nombreuses pour les années 1951 et 1955 (tableau 1), celles qui ont une "coloration suburbaine», donc qui vendent différents biens de consommation en les mettant en scène dans un environnement suburbain, sont beaucoup plus fréquentes.

32. Voir The Gazette, 6 janvier 1951, p. 16; The Gazette, 29 février 1959, p. 21. 
fréquemment écho aux événements et aux tendances associées à la massification du processus de suburbanisation, qu'il s'agisse de cahiers spéciaux à l'occasion de la semaine nationale de l'habitation, où le bungalow tient le premier rôle ${ }^{33}$, ou encore d'articles faisant un lien étroit entre natalité et étalement urbain dans la région métropolitaine ${ }^{34}$.

Différentes entreprises vont également associer leurs produits au mode de vie et à l'environnement suburbain. Les exemples sont nombreux et vont de la distillerie Calvert, qui organise au milieu des années 1950 un concours international d'architecture pour trouver la «Maison Canadienne de Demain ${ }^{35}$ ", aux pleines pages qu'achète Simpson's pour vendre des accessoires de patio qui sont représentés dans un décor tout ce qu'il y a de plus banlieusard $^{36}$. La terminologie suburbaine elle-même est utilisée pour identifier certains produits et les associer à cet environnement. C'est par exemple le cas des clôtures Frost, qui deviennent la «clôture Frost suburbaine» dans une publicité qui joue sur la question de la sécurité des enfants qui risquent de s'aventurer dans la rue ${ }^{37}$. C'est également le cas des pneus «Suburbanite» produits par Goodyear ${ }^{38}$. Mentionnons enfin cette publicité de la Montreal City and District Bank, parue en 1965, et invitant les lecteurs de The Gazette à profiter pleinement du congé que leur offre la fête du Travail (figure 2). S'adressant aux "millions of hardworking men and women", elle met en scène un homme dormant dans un hamac, dans le jardin de sa résidence, dans un décor qui évoque beaucoup plus Pointe-Claire que Pointe-SaintCharles. Elle permet de constater à quel point le mode de vie et l'environnement suburbain apparaissent, pour ceux qui conçoivent ces publicités, comme le mode de vie "par défaut» des travailleurs de l'époque, ou du moins celui auquel ils devraient aspirer.

33. Voir La Presse, 26 septembre 1959, p. 17-21.

34. "The thousands of new families brought a growing demand for new homes. They wanted their own plot of land and home of their own. They got what they were looking for - in suburbia ", Bob Hayes, "Children... Stimulus for Building Urban Sprawl», The Gazette, 30 mai 1959, p. 31.

35. Le concours organisé par Calvert's Distillers Ltd., en collaboration avec l'école d'architecture de l’Université McGill, est publicisé tout au long de l'année 1955 dans les journaux. Voir, par exemple, $L a$ Presse, 5 mars 1955 , p. 62. Le Centre canadien d'architecture a consacré une exposition en ligne à ce concours: "Concours Calvert House pour la maison canadienne de demain ", Centre canadien d'architecture, $<$ http: / / www.ccc.umontreal.ca / fiche_concours.php?lang=fr\&cId $=241>$.

36. The Gazette, 26 mai 1951, p. 6.

37. La Presse, 21 mai 1959, p. 23.

38. La publicité «Le temps est aux Suburbanite» ne fait pourtant aucune référence directe à la banlieue. La Presse, 28 novembre 1959, p. 8. 
Figure 2

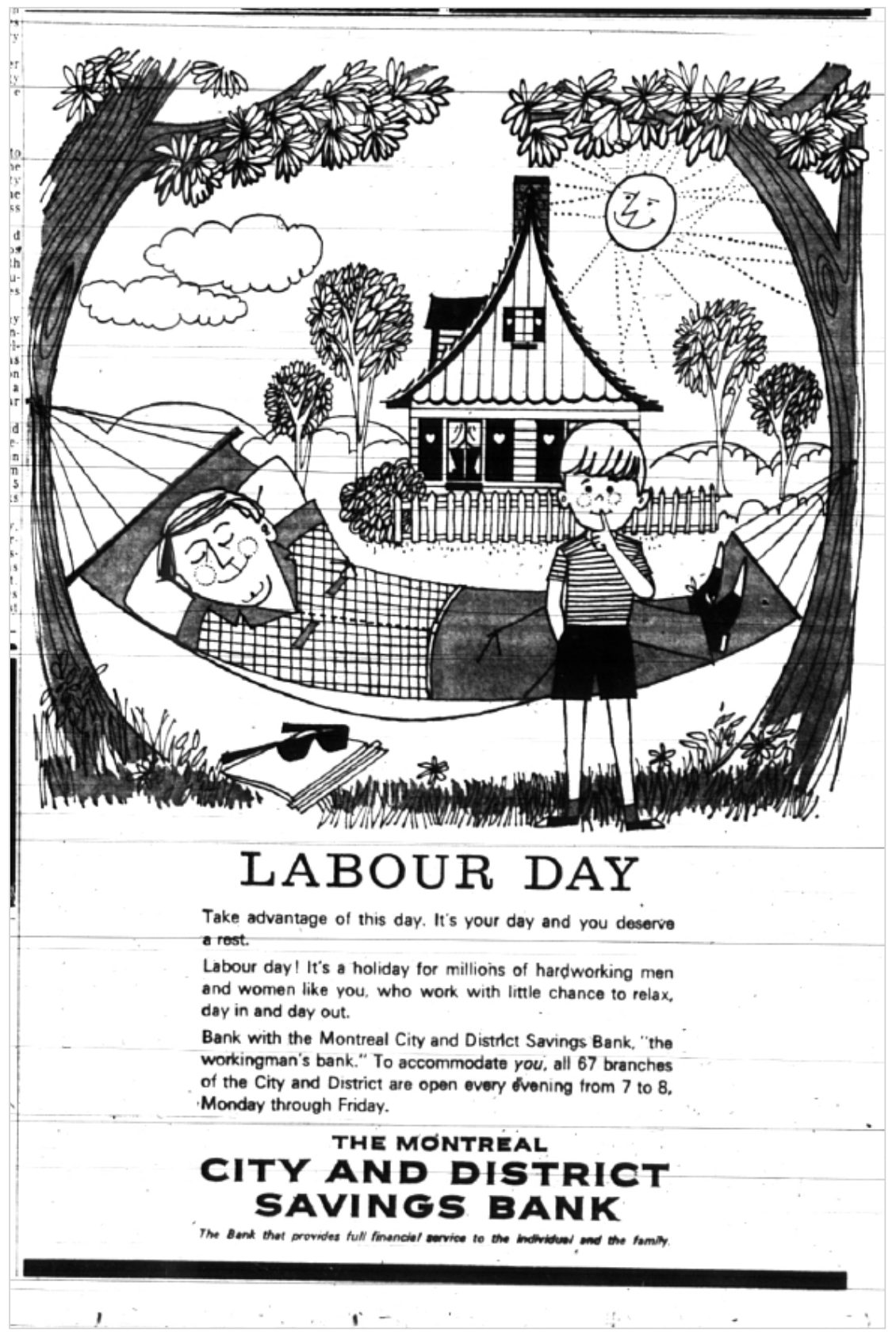

«Labour Day », le mode de vie suburbain comme mode de vie par défaut de l'ouvrier des années 1960 (The Gazette, 4 septembre 1965, p. 4). 
Figure 3

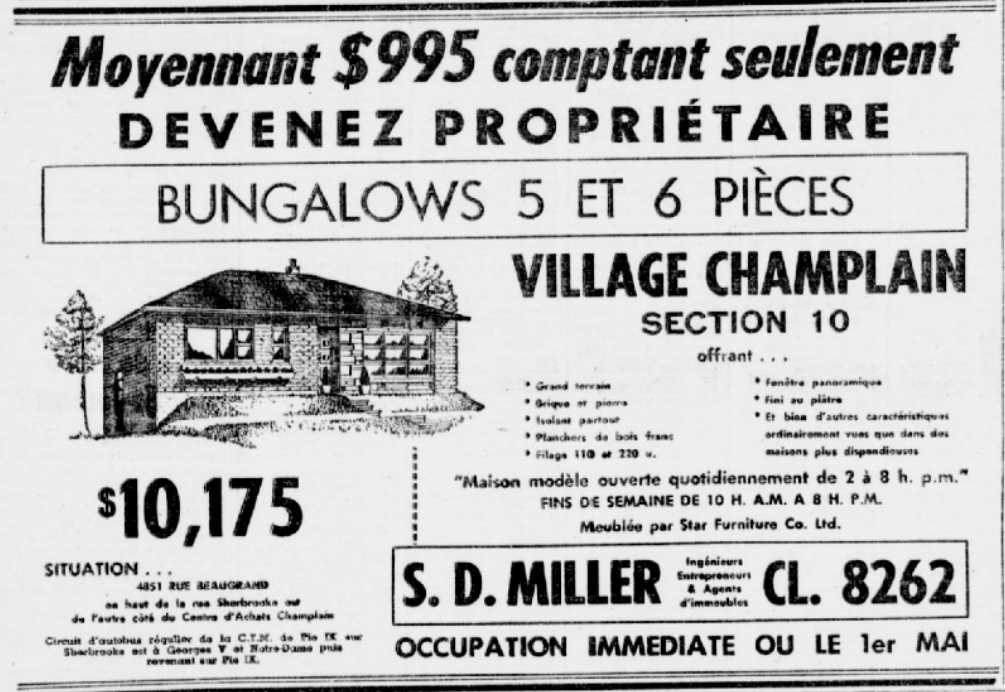

«Moyennant $\$ 995$ comptant seulement, DEVENEZ PROPRIÉTAIRE»: un exemple de la publicité type (La Patrie, 16 avril 1955, p. 58).

\section{UNE PUBLICITÉ TYPE}

Pour convaincre les lecteurs et les lectrices des grands quotidiens montréalais d'acheter une résidence unifamiliale détachée dans une des banlieues qui se multiplient dans la région montréalaise, les promoteurs immobiliers vont se tourner vers une publicité type dont les caractéristiques de base varient assez peu durant la période étudiée - et au-delà, si on se fie aux recherches de Gérald Fortin ${ }^{39}$. Cette publicité type, on la retrouve dans les journaux anglophone et francophones, et durant toute la période étudiée. On y retrouve: une illustration représentant le ou les modèles de maison proposés, le nom du développement et les indications pour s'y rendre (parfois accompagnées d'une carte rudimentaire), les coordonnées du promoteur, une liste de caractéristiques de la maison et, surtout, son prix (figure 3). Cette description correspond à la grande

39. Fortin explique à ce sujet que «[1]’annonce type des années 1956-1970 met l'accent sur le prix et indique comment se rendre sur le lieu de la construction. On insistera à l'occasion sur les équipements et les accessoires et on invoquera la nature.» Mes recherches contredisent toutefois cette dernière phrase: dès le début des années 1950, on insiste presque systématiquement sur les accessoires et les équipements de la maison, et la nature, bien que présente, est loin d'être un thème dominant. G. Fortin, La publicité sur le logement neuf..., p. 55. 
majorité des publicités recensées durant la période 1951-1969 et est révélatrice du fait que l'achat d'une maison est d'abord et avant tout, pour le ménage montréalais moyen des Trente Glorieuses, la plus importante transaction financière à laquelle il sera confronté. Si l'on examine de plus près cette publicité type, il est possible de faire ressortir les grandes stratégies utilisées par les promoteurs dans ce contexte.

Soulignons tout d'abord l'importance de ce que j'appellerais «l'appel à la modernité». Le qualificatif «moderne», et ses dérivés, est utilisé de manière abondante dans les publicités recensées pour décrire les habitations proposées et le mode de vie qui leur est associé. Ce n'est ni entièrement inattendu ni entièrement nouveau: dès le début du $\mathrm{XX}^{\mathrm{e}}$ siècle, la supériorité technique des infrastructures et des maisons suburbaines est mise de l'avant pour faire la promotion de banlieues destinées aux classes aisées $^{40}$. Ce qui change durant cette période, c'est l'intensité avec laquelle ce vocabulaire de la modernité domestique est déployé. Dès 1951, on parlera par exemple de bungalows conçus pour le «modern living in a private residential development - scientifically planned, with newest most modern features ${ }^{41} »$. Cette surenchère se poursuit tout au long de la période, avec des maisons «ultra-modernes» ou des développements «à l'avant-garde du progrès ${ }^{42}$. Dans le même esprit, les longues listes de caractéristiques qui accompagnent généralement ces publicités permettent de présenter aux futurs acheteurs ce qui constitue concrètement la modernité suburbaine et jouent fortement sur l'idée d'un mode de vie auquel l'on n'aurait d'autre choix que de se joindre, comme dans cet exemple de 1955 :

Abri d'auto - La façon la plus pratique que l'on puisse trouver au Canada et aux États-Unis pour remiser l'auto.

Chauffage à l'eau chaude - Le seul chauffage désirable pour votre maison au Canada.

Grande cuisine donnant directement sur la salle à manger qui donne sur le salon, créant ainsi une grande pièce à l'atmosphère familiale tant prisée ici à Montréal. Ventilateur électrique.

Tuiles et garnitures de couleur dans la salle de bain.

40. H. Bérubé, Des sociétés distinctes..., p. 108-116, 166-173.

41. The Gazette, 23 juin 1951, p. 2.

42. Voir La Patrie, 10 septembre 1955, p. 51; La Presse, 29 août 1959, p. 23. 
Murs de plâtre - que des milliers de gens préfèrent aux autres matériaux de remplacement quant à la résistance ${ }^{43}$.

Un autre élément important qui revient presque systématiquement dans les publicités recensées est la référence à ce que j’appellerais la « sainte trinité suburbaine». La présence et la proximité de trois institutions vont apparaître comme incontournables dans la mise en marché de bungalows: les écoles, les églises et les centres commerciaux (figure 4 : à consulter en ligne). Les premières témoignent de la centralité de la famille et des jeunes enfants dans l'idéal suburbain, alors que les secondes nous rappellent que la société montréalaise des Trente Glorieuses est loin d'avoir tourné le dos à la pratique religieuse ${ }^{44}$. Quant aux centres commerciaux, leur développement est consubstantiel à celui des banlieues d'après-guerre.

Le dernier élément relatif aux grandes stratégies déployées par les publicitaires est ce qu'on pourrait appeler globalement la question d'argent, qui nous ramène à l'importance de la transaction que représente l'achat d'une maison. Les publicités analysées ne sont pas sans le rappeler fréquemment d'ailleurs. Par exemple, dans La Presse, une série de publicités est accompagnée d'un article du quotidien intitulé (en gros caractères) «L'achat d'une maison est une affaire sérieuse ${ }^{45}$ ». Plus spécifiquement, lorsque les publicités traitent du prix des résidences proposées aux lecteurs, on note une rhétorique peu étonnante sur le bas prix des maisons et sur le comptant limité qu'il faut verser pour avoir accès à la propriété. Plus intéressante est la grande fréquence d'indices relatifs aux craintes des acheteurs face à d'éventuels frais cachés. Les publicitaires vont souvent aller au-devant de ces inquiétudes pour rassurer les consommateurs, suggérant indirectement qu'il s'agissait d'une pratique assez courante. Un exemple frappant de cette réalité est la publicité pleine page publiée dans The Gazette en 1961 pour un développement de Chambly. On y insiste sur l'absence de frais cachés et on propose même au lecteur une vingtaine de questions que tout acheteur devrait poser avant d'acheter une maison ${ }^{46}$.

43. La Patrie, 4 juin 1955 , p. 49.

44. Soulignons tout de même que l'église commencera à disparaître de certaines des publicités anglophones et francophones à partir du milieu des années 1960.

45. La Presse, 26 septembre 1959, p. 25.

46. "At Chambly West, the price we say is the price you pay. There are NO HIDDEN CHARGES.» La publicité contient une section très détaillée sur les frais qui auraient possiblement pu être cachés, mais qui sont mis ici en relief pour bien montrer l'honnêteté du promoteur. The Gazette, 2 décembre 1961, p. 14 (souligné dans l'original). Une publicité très similaire, pour un développement à Brossard, est publiée dans La Presse, 12 juin 1965, p. 22. 
Figure 5

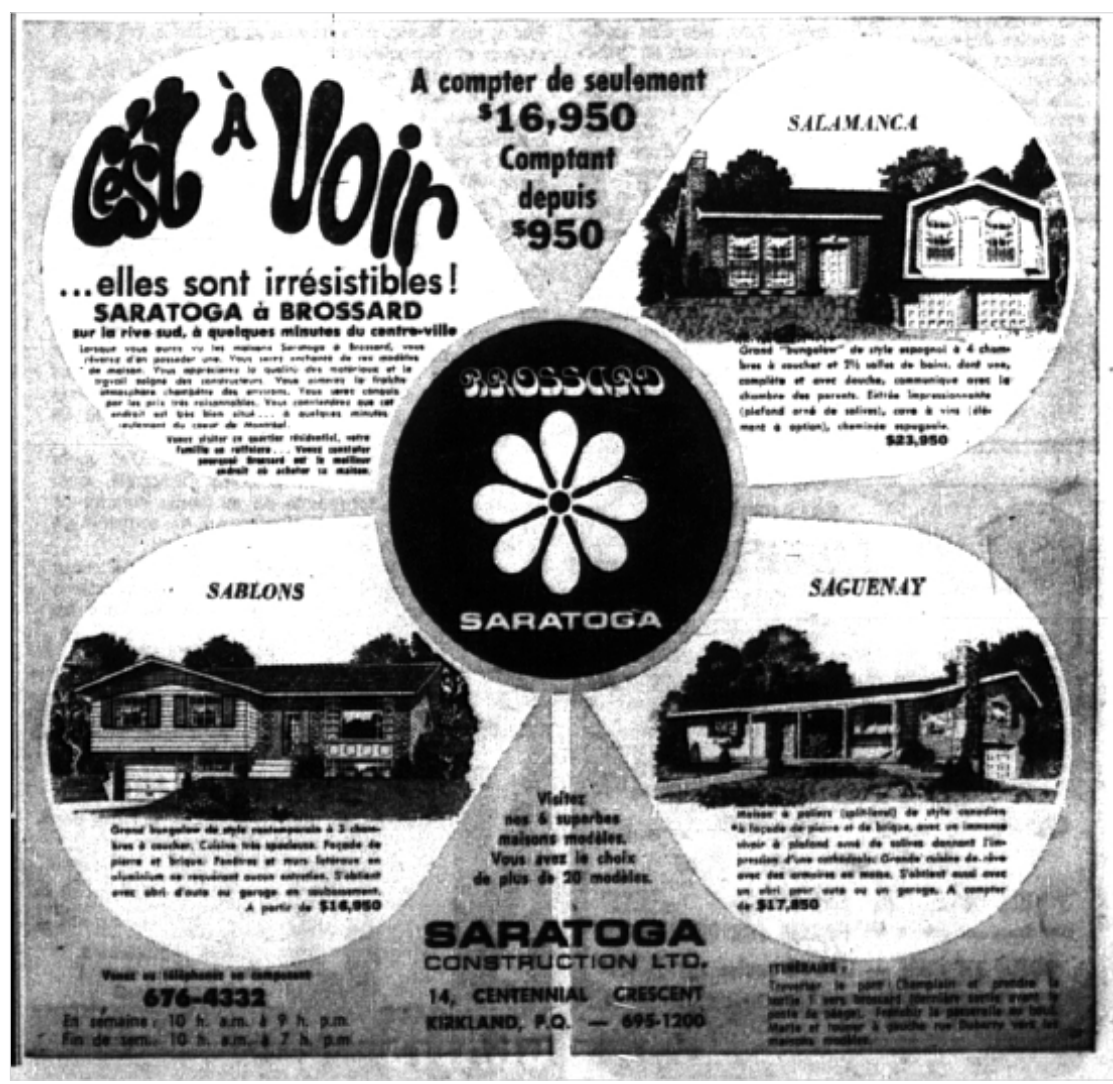

«C'est à voir... elles sont irrésistibles!»: publicité au graphisme un peu plus audacieux, mais dont les caractéristiques de bases sont assez classiques (La Presse, 26 juillet 1969, p. 50).

Ces caractéristiques et ces stratégies forment la fondation sur laquelle s'appuie une large majorité des publicités recensées et analysées. Elles témoignent d'une grande stabilité, voire d'une homogénéité des discours publicitaires véhiculés durant la période étudiée dans les grands quotidiens montréalais de langue française ou anglaise. Pour les publicités qui se conforment à ce modèle, la principale différence d'une décennie à l'autre est probablement au niveau du graphisme, qui s'adapte au goût du jour, comme le montre cette publicité pour un développement de Brossard qui a un petit quelque chose de psychédélique (figure 5). 


\section{DE LA MODESTIE AU GRAND LUXE}

Si la publicité type décrite dans la section précédente domine les pages des grands quotidiens montréalais, elle ne résume pas à elle seule le discours qui se déploie à l'époque pour vendre des maisons aux habitants de la métropole. En fait, au cours des deux décennies étudiées, une part grandissante des publicités produites va varier discours et approches pour convaincre les consommateurs. Cette tendance générale semble refléter à la fois un raffinement des stratégies publicitaires, mais aussi un élargissement de la clientèle à laquelle on s'adresse, élargissement qui ouvre la porte à des publicités plus ciblées. Ainsi, alors qu’on associe généralement les années 1950 aux Trente Glorieuses la première chose qui frappe lorsqu'on se penche sur les pages publicitaires des journaux durant cette décennie, c'est l'impression d'une certaine retenue dans le déploiement de la société de consommation. Retenue qui nous rappelle que certaines des restrictions relatives au conflit mondial sont encore en vigueur ou font encore sentir leurs effets, et que la région montréalaise demeure en proie à une véritable crise du logement ${ }^{47}$. Un bon exemple de cette retenue est le fait que les publicités liées au monde de l'automobile insistent presque systématiquement sur la faible consommation des modèles proposés ${ }^{48}$.

Les publicités relatives aux maisons unifamiliales détachées en banlieue de Montréal font écho à cette réalité. L’un des premiers exemples que nous avons relevés en témoigne éloquemment (figure 6). La "nouvelle maison CUMMINGS AMÉLIORÉE» qui apparaît sur cette publicité et de taille et de facture modestes. La maison modèle que propose le promoteur aux éventuels acheteurs est décorée par Dupuis Frères, «le magasin du peuple", situé dans l'est de la ville et on indique comment s'y rendre en autobus, assumant que l'acheteur potentiel ne dispose pas de cette voiture qu'on associera si étroitement au mode de vie suburbain. Le publicitaire précise: «Vous serez émerveillés de voir le confort et la dignité que vous offre à si bon prix, la conception moderne et avancée de cette demeure ${ }^{49}$." Cette idée du luxe à petit prix est une stratégie assez classique dans le monde publicitaire. Ce qui est notable en 1951, c’est qu'elle est généralisée

47. Amélie Bourbeau, «"Tuer le taudis qui nous tuera”: crise du logement et discours sur la famille montréalaise d'après-guerre (1945-1960)", mémoire de maîtrise (histoire), Université de Montréal, 2002, $149 \mathrm{p}$.

48. La Studebaker Champion 1951 est ainsi d'abord présentée comme "économique» et n'ayant "[p]as besoin de carburant cher», La Patrie, 3 février 1951, p. 42. Une publicité de Ford, reproduite en français et en anglais la même année, vante la faible consommation d'essence de ses voitures. The Gazette, 24 février 1951, p. 7 ; La Patrie, 3 mars 1951, p. 61.

49. La Patrie, 14 avril 1951, p. 62. 
Figure 6

62

\section{ENFIN! VOUS POUVEZ VISITER la. nouvelle MAISON CUMMINGS AMÉLIORÉE}

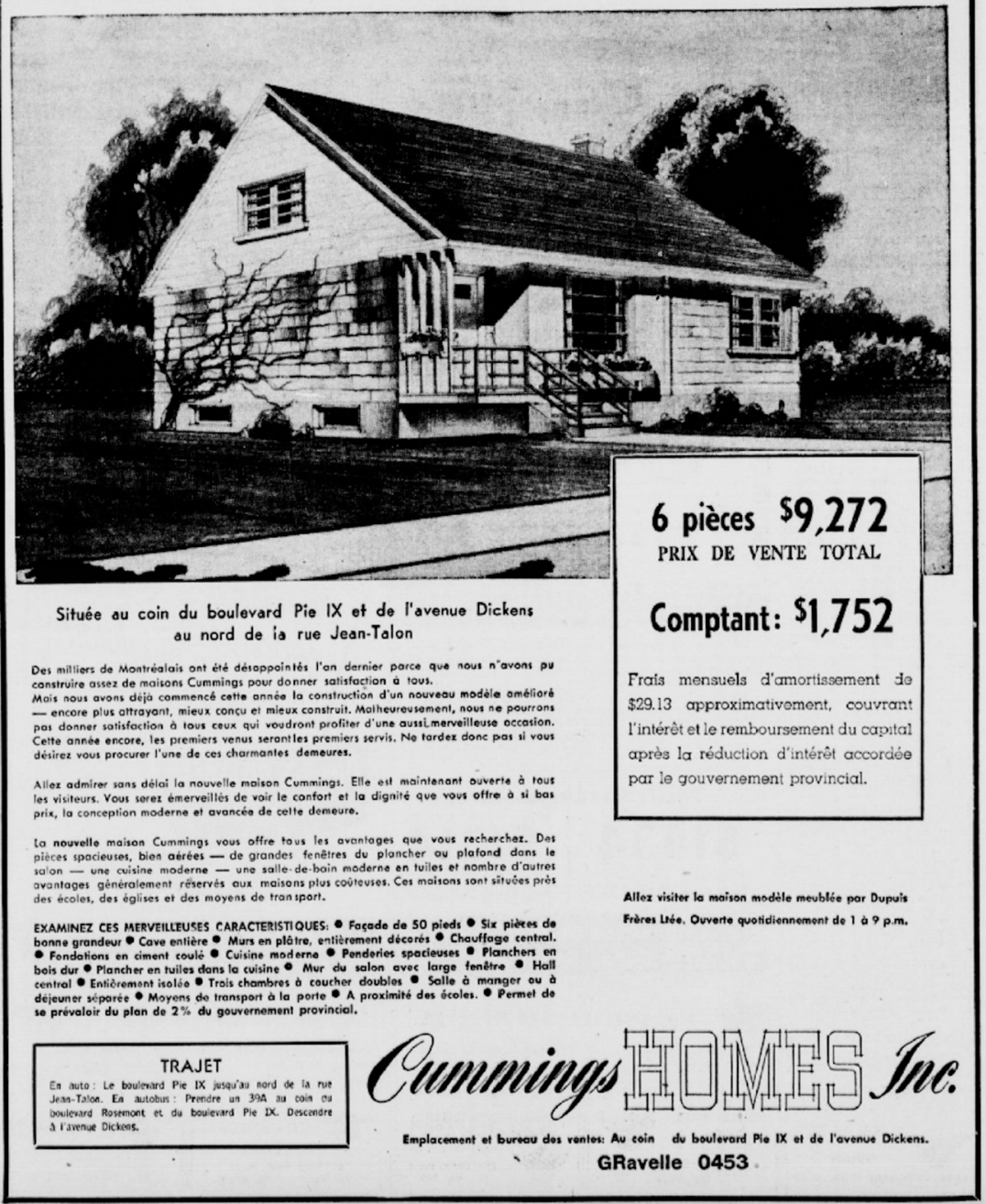

«ENFIN! VOUS POUVEZ VISITER la nouvelle maison CUMMINGS AMÉLIORÉĖ : un exemple de la modestie des maisons proposées aux acheteurs au début des années 1950 (La Patrie, I4 avril 195I, p. 62). 
et qu'elle ne coexiste pas avec une offre qui serait destinée à une clientèle plus aisée ${ }^{50}$.

C'est une tendance qui est toujours perceptible au milieu des années 1950. Par exemple, dans une publicité pour le Village Champlain, un assez vaste développement situé dans le quartier actuel d'Hochelaga-Maisonneuve ${ }^{51}$, on indique qu'il "n'y a pas de pierre que nous n'ayons remuée pour vous procurer ce qu'il y a de plus opulent». Cependant, les bungalows proposés sont, encore une fois, de taille et de facture modestes, bien que l'on indique qu'ils ont des «caractéristiques ordinairement vues que dans les maisons plus dispendieuses ${ }^{52}$. C'est aussi le thème utilisé dans une publicité pleine page - pratique que l'on voit plus fréquemment à partir de 1955 - pour le quartier Meadowbrook de Saint-Vincent-de-Paul : si on explique que les maisons ont été dessinées "par un bureau d'architectes renommé», on précise qu'elles sont «à un prix que vous pouvez payer $"^{53}$. La presse anglophone véhicule un discours publicitaire similaire. Par exemple, le Fortin Project de Sainte-Rose propose des bungalows à «an extremely sensible price ${ }^{54}$ ».

Cette modestie est toujours perceptible dans les années 1960, mais elle coexiste alors avec des projets plus élaborés et fastueux. Un bon exemple de cette évolution est la modernisation des publicités mises de l'avant par le promoteur Parkview en 1960 (figure 7 : à consulter en ligne). On note immédiatement la facture plus élaborée du graphisme, le prix assez élevé des maisons (entre 22975 \$ et $25475 \$$ ), mais surtout un langage qui met plus clairement l'accent sur le luxe $\mathrm{e}^{55}$. On parle de «luxueux split-levels» et surtout de «l'exclusif district domiciliaire Persillier d'Ahuntsic». D’ailleurs, la même journée, Parkview Construction Ltd publie, à la page précédente de La Presse, une publicité plus modeste pour des maisons à Saint-Vincentde-Paul à un prix nettement moins élevé (14 995\$) et avec un discours plus sobre. Visiblement, le promoteur a maintenant les moyens et l'ambition de courtiser plusieurs clientèles à la fois. C'est une évolution que

50. Il va de soi que cette clientèle plus aisée cherche également à se loger à l'époque, mais elle n'a probablement pas besoin de ces publicités pour savoir où se loger et à quelles conditions. H. Bérubé, Des sociétés distinctes..., p. 173-182.

51. À son sujet, voir notamment Mathieu Pomerleau et Daniel Sainte-Marie, Le village Champlain. Étude historique et typo-morphologique en prévision du plan d'implantation et d'intégration architecturale (PIIA) (Montréal, Ville de Montréal [arrondissement Mercier-Hochelaga-Maisonneuve], 2014.

52. Ici aussi, on indique à l'acheteur potentiel comment se rendre sur place en autobus. La Patrie, 2 avril 1955, p. 44.

53. La Patrie, 4 juin 1955 , p. 49.

54. On y parle également de "very low taxes». The Gazette, 10 septembre 1955, p. 4.

55. La Presse, 29 octobre 1960, p. 45. 
l'on note aussi dans la presse anglophone. Ainsi, en 1960, on fera la promotion des maisons de type "Aristocrat» des Westchester Estates à Beaconsfield (qui se vendent au prix très élevé pour l'époque de $38000 \$$ ); des «exclusive homes» de Rideau Park à Brossard (25 250\$); sans parler de «Laurier Court» à Beaconsfield, que l'on décrit comme «[the] correct address on the Lakeshore $»^{56}$. En 1965, différentes publicités poussent un peu plus loin sur ce front, associant l'achat d'une maison à un indéniable embourgeoisement. C'est le cas par exemple de Greendale, «the new executive community in Pierrefond and Dollard des Ormeaux» où l'achat d'une maison est accompagné d'une «Private Marina and Country Club with swimming pools ${ }^{57}$. Cette référence est encore plus explicite dans une publicité pour la Terrasse des Érables, à Montréal-Nord, dont les maisons sont décrites comme "conçues pour la classe bourgeoise ${ }^{58}$ ".

Cette diversification relative des clientèles visées se fait en parallèle avec la transition des petits promoteurs aux grandes entreprises qui gèrent de multiples développements aux quatre coins de la région métropolitaine. Ainsi, alors que la presque totalité des publicités recensées dans les années 1950 se conforment au premier modèle (un petit promoteur et un petit nombre de maisons à vendre), on voit apparaître, dans les années 1960, de plus en plus d'entreprises du deuxième genre. Parkview Construction Ltd en est un bon exemple. Dès 1960, l'entreprise fait la promotion de maisons sur quatre sites distincts: Sainte-Geneviève, Châteauguay, Saint-Vincent-dePaul et Ville Saint-Laurent (dans ce dernier cas, il s'agit de duplex) ${ }^{59}$.

Ajoutons que cette courbe n'est pas qu'ascendante. À la fin des années 1960 , on peut clairement sentir, dans les discours publicitaires, l'essoufflement du dynamisme économique lié aux Trente Glorieuses, et tout particulièrement la hausse importante des taux d'intérêt qui marque la fin de la décennie $^{60}$. Ainsi, alors qu'il était fréquent de retrouver, dans les publicités de la fin des années 1950 et du début des années 1960, un argumentaire voulant que l'achat d'une maison soit, sur le plan financier, nettement plus

56. Ces publicités apparaissent toutes à la même page de The Gazette, 22 avril 1961, p. 39.

57. The Gazette, le 16 janvier 1965, p. 39.

58. La Presse, 21 août 1965, p. 6.

59. On informe d'ailleurs le lecteur que l'on «remarque aujourd'hui des maisons PARKVIEW dans tous les districts de Montréal, offrant un choix considérable et varié de maisons de qualité à des prix très attrayants ", La Presse, 15 octobre 1960, p. 55.

60. Sur les inquiétudes relatives à l’inflation au Québec, voir S. O’Neill, «L’argent ne fait pas le bonheur...", p. 111-131. 
avantageux que la location d'un appartement ${ }^{61}$, un certain nombre de promoteurs vont commencer à inverser ce raisonnement. Alors que certaines publicités invitent l'acheteur à se presser avant que les taux d'intérêt rendent l'acquisition d'une propriété impossible ${ }^{62}$, d'autres, comme Belcourt Homes, informent le lecteur qu'avec la hausse brutale des taux d'intérêt, acheter devient impossible. L'entreprise offre donc, en attendant des jours meilleurs, des locations ${ }^{63}$. Bref, les promoteurs et les publicitaires s'adaptent assez rapidement à un contexte économique changeant.

\section{DE LA MAISON À L'ENVIRONNEMENT}

Une autre des transformations importantes que l'on note durant cette période est le passage d'une publicité centrée uniquement sur la maison à un discours qui valorise de plus en plus l'environnement dans lequel s'insère cette résidence. Ainsi, la publicité type dont j’ai parlé plus tôt est généralement centrée sur la maison et ses caractéristiques mais, dès le milieu des années 1950, un petit nombre de cas met l'accent sur les qualités de l'environnement où se trouve cette demeure. À la fin des années 1950, cette nouvelle préoccupation des promoteurs semble d'abord et avant tout répondre à la crainte de ce que l'on va appeler, dans les publicités anglophones, le "pioneering", c'est-à-dire le fait de faire partie de ces premiers acheteurs - les "pionniers " - qui vont s'installer dans des développements où tout est encore à faire. Par exemple, dans une publicité pour les Jardins Duvernay, on souligne à grands traits que des sites ont déjà été réservés pour les principaux bâtiments institutionnels ${ }^{64}$. Dans le cas du «Domaine du Pont Champlain», on précise qu'il n'y aura "[aucune] attente pour les services urbains au Domaine du Pont Champlain [et que le] pavage des rues se fait actuellement et tous les services sont en voie d'installation ${ }^{65}$ ». Dans une publicité élaborée pour le projet «Treasure Island on Île Perrot» (figure

61. Une publicité pour les maisons du Domaine Bellerive demande ainsi à l'acheteur: «Pourquoi payer loyer?", La Presse, 14 mars 1959, p. 29. Une autre publicité, pour un développement de Repentigny, apostrophe le lecteur ainsi : “Capitalisez au lieu de payer du loyer!», La Presse, 18 juin 1960, p. 9.

62. Une publicité pour Candiac explique: "Every day you delay makes home ownership more expensive. There is no better time than now ", The Gazette, 22 février 1969, p. 28.

63. "[At] today's exorbitant mortgage rates, buying is absolutely out of the question for most people - except maybe millionaires. That is why Belcourt has restructured its entire home-building operation for rentals rather than sales. This allows you to delay your final decision on home purchase till things get better (if they ever do)", The Gazette, 25 janvier 1969, p. 24. Une publicité pour l'île des Sœurs fait de même: "Do you want to pay reasonable rent or buy a house and pay high taxes, a high down payment, and a high interest rate?», The Gazette, 22 février 1969, p. 31.

64. La Presse, 11 avril 1959, p. 7.

65. La Presse, 9 janvier 1965, p. 7. 
Figure 9

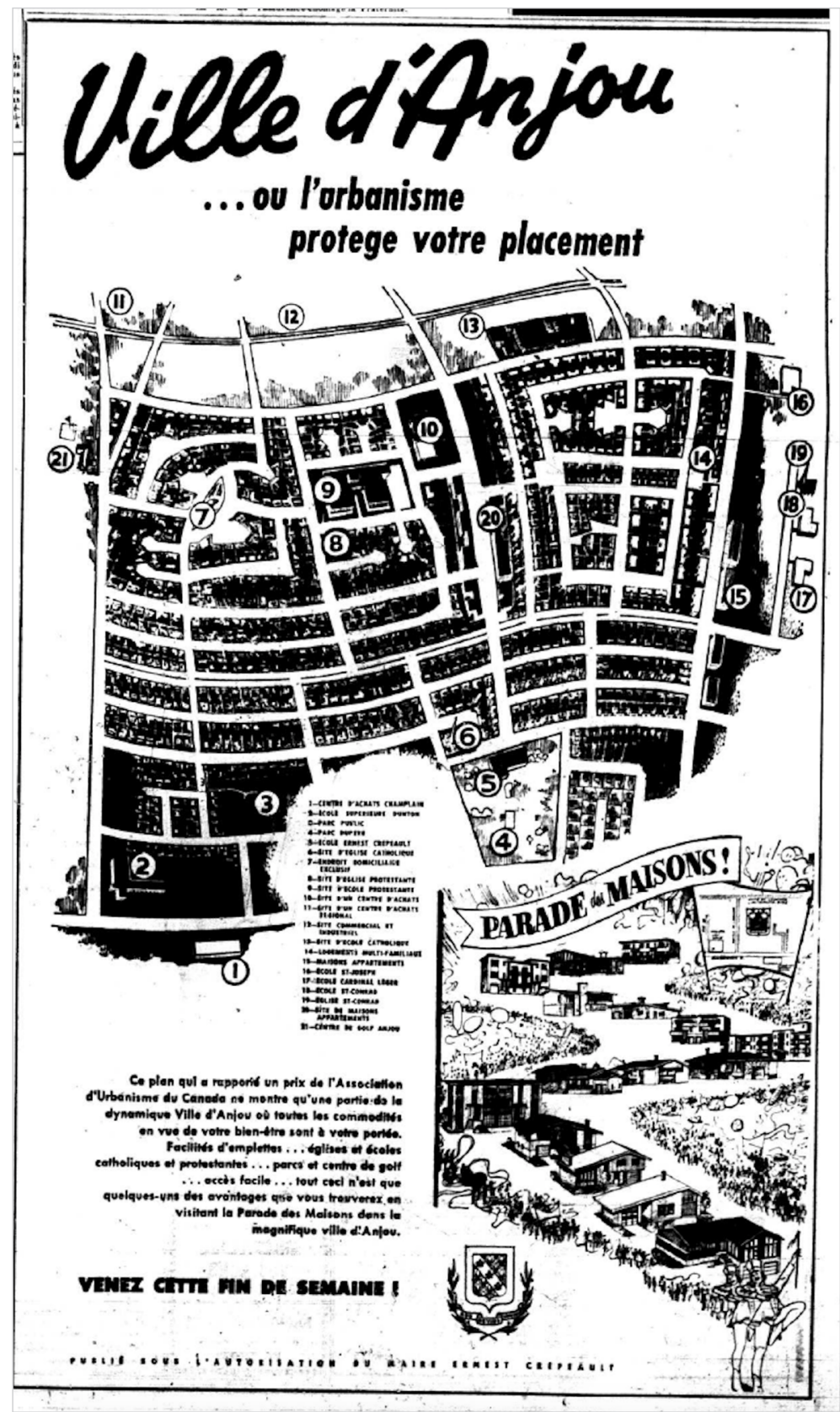

«Ville d'Anjou... où l'urbanisme protège votre placement»: une des publicités produites par la municipalité de Ville d'Anjou dans le cadre de sa campagne de 1959 (La Presse, I4 mars 1959, p. I3). 
8 : à consulter), on propose au lecteur une section question/réponse où l'on fait explicitement référence à cette notion de "pioneering»:

Q. What makes Treasure Island on Île Perrot such a desirable place to live in?

A. Because it is a complete, planned, residential community with every convenience... far enough away for peace and quiet yet with easy and fast access to Montreal.

Q. What do you mean by a "complete, planned community"?

A. A community that is all planned beforehand. Planned with churches, schools, shopping, recreation areas, everything actually in the community. Curved streets, cul-de-sacs, crescents are typical of such an area. Sidewalks, paved roads, sewers, streetlighting are all in. There is no need for pioneering $g^{66}$ !

À la fin des années 1950, on retrouve dans les pages de La Presse une offensive publicitaire qui va dans ce sens, mais qui se distingue pour trois raisons: d'abord, sur le plan du graphisme, ces publicités sont particulièrement élaborées; ensuite, elles sont publiées non pas à l'initiative d'un promoteur, mais à celle de la mairie de Ville d'Anjou; enfin, elles sont les premières à proposer un argumentaire aussi élaboré sur l'importance de l'environnement suburbain. La première publicité de cette série est publiée en février ( sous l'autorisation du maire Ernest Crépeault ${ }^{67}$ ), mais il faut attendre la deuxième pour voir apparaître cet argumentaire (figure 9). On y affirme que «l'urbanisme protège votre placement», car la ville se serait dotée d'un plan de développement qui aurait remporté un prix de l'Association d'urbanisme du Canada. La carte qui domine cette publicité permet notamment de localiser l'emplacement d'écoles, de parcs et de centres commerciaux présents ou à venir dans la municipalité ${ }^{68}$. D’autres publicités, toutes aussi élaborées, suivront au long de l'année, comme celle du 21 mars qui présente la municipalité comme la "ville modèle la plus progressive de l'île de Montréal» ou encore celle du 11 avril suivant qui explique que la municipalité aurait évité «les problèmes d'une ville jeune» et que les services y sont au rendez-vous ${ }^{69}$.

Durant la période étudiée, aucune autre municipalité n’ira aussi loin, mais on observe, dans les années 1960, un nombre croissant de publicités ponctuelles émanant directement de gouvernements municipaux suburbains. On n'y vend évidemment pas de bungalow, mais on cherche à

66. The Gazette, 23 janvier 1965, p. 29.

67. La Presse, 28 février 1959, p. 12.

68. La Presse, 14 mars 1959, p. 13.

69. La Presse, 21 mars 1959, p. 13 et 11 avril, p. 13. 
guider l'acheteur vers la «bonne municipalité», qu'il s'agisse de cette publicité qui explique que «Roxboro jouira de services aussi nombreux et aussi avantageux, sinon plus, que toute autre municipalité de la périphérie de Montréal» ou alors de celle qui explique que, avec la construction à venir du tout nouveau pont Champlain, le "destin a choisi » Brossard $^{70}$. Ces publicités témoignent certainement de la concurrence de plus en plus vive entre les différentes municipalités suburbaines de la région de Montréal pour attirer, chez eux, acheteurs de maisons et futurs contribuables.

\section{DE L'ENVIRONNEMENT À LA COMMUNAUTÉ}

Dans son ouvrage sur la publicité et la classe moyenne, Sébastien Couvrette souligne que le discours véhiculé fait d'abord et avant tout la "promotion des valeurs individualistes ${ }^{71} »$. C'est certainement le cas ici, mais cet individualisme a ses limites. Surtout dans les années 1960, il est articulé, dans un nombre croissant de publicités destinées à une clientèle plus aisée, à une préoccupation pour l'appartenance à la (bonne) communauté. C'était déjà une des pistes empruntées dans ces publicités de Ville d'Anjou en 1959, l'une d'entre elles portant le titre «Votre genre de voisin à Ville d'Anjou» et expliquant que la municipalité est habitée par des gens respectables, une respectabilité associée à l'occupation de ces individus: "médecins, ingénieurs, professionnels $»^{72}$. À partir du début des années 1960 , on verra de plus en plus fréquemment apparaitre cette idée.

C'est particulièrement le cas de la toute nouvelle municipalité de banlieue de Lorraine, créée en 1960. Dans de somptueux "publireportages", son promoteur, la Lorraine Development Corporation, propose un argumentaire de vente élaboré qui met l'accent sur les six années passées à planifier et à préparer cette communauté. La cohésion sociale qui en résulterait combinerait l'esprit métropolitain et la vie de campagne, tout en étant une garantie pour ce qui est de la valeur des propriétés qui s'y trouvent ${ }^{73}$. C'est une offensive publicitaire qui se poursuit tout au long des années 1960, et dans le cadre de laquelle la maison est aisément éclipsée par tout ce qui l'entoure, qu'il s'agisse des avantages du site, de son aspect planifié et contrôlé par une commission architecturale («an award winning community ") ou encore des différentes activités qui sont

70. La Presse, 4 avril 1959, 21 et 2 mai 1959, p. 61.

71. S. Couvrette, Le récit de la classe moyenne..., p. 189-190.

72. La Presse, 18 avril 1959, p. 23.

73. The Gazette, 19 août 1961, p. 7. 
offertes dans son centre communautaire («how Lorraine doubles as a "classroom») ${ }^{74}$.

Différents promoteurs imiteront l'exemple proposé par Lorraine et contribueront à nourrir cette importance croissante de la communauté dans laquelle s'insèrent les maisons proposées aux acheteurs. Dans plusieurs cas, l'argumentaire demeure en bonne partie financier - il s'agit de protéger la valeur de la propriété. C'est le cas de cette modeste publicité pour Monarch Construction qui résume bien l'affaire: "A lovely house becomes a lovely home in the right neighbourhood ${ }^{75}$.» C'est également le cas de publicités pour le développement Sainte-Thérèse-en-Haut qui parlent, comme dans le cas de Lorraine, d'une «award winning community ${ }^{76}$ ", et qui mettent l'accent sur la localisation de la maison:

The location of your home is just as important as the house itself... probably even more important. As the years go by, you may wish to expand, add to or improve your home... but there's nothing you can do about its location. You can't expand its prestige, add to its convenience or improve its surroundings. That's why it's so important to look ahead to tomorrow when you buy a home today ${ }^{77}$ !

Mais un nombre croissant de publicités ira plus loin en mettant de l'avant l'environnement socioprofessionnel auquel se joindra l'acheteur potentiel. Professionnels et hommes d'affaires deviennent alors, comme on l'a vu plus tôt pour Ville d'Anjou, des voisins désirables, synonymes d'un environnement sain et d'un investissement prometteur. C'est le message que transmet cette publicité pour Val des Arbres à Duvernay, qui souligne «PROFESSIONNELS, EXÉCUTIFS [sic], HOMMES D’AFFAIRES - Venez vivre avec des gens de votre milieu - dans une maison si NOUVELLEMENT différente que vous ferez l'envie de votre entourage ${ }^{78}$ »; ou encore cette publicité pour Laurier Park qui explique: «If I buy a house in Laurier Park what kind of neighbors will I have? Doctors, Lawyers, Architects, Scientists, Engineers $^{79}$.»

\section{LA FAMILLE NUCLÉAIRE AU CCEUR DE L'IDÉAL SUBURBAIN?}

De la maison et ses caractéristiques techniques, on est donc passé - dans certaines des publicités recensées du moins - à l'environnement physique

\footnotetext{
74. The Gazette, 20 février 1965, p. 27 et 28 août 1965, p. 27.

75. The Gazette, 20 février 1965, p. 26.

76. The Gazette, 20 mars 1965, p. 27.

77. The Gazette, 3 avril 1965, p. 31.

78. La Presse, 16 janvier 1965, p. 31.

79. The Gazette, 1 mai 1965, p. 34.
} 
et social dans lequel s'inscrira cette résidence et ses habitants. Et c'est vers ces nouveaux banlieusards que je voudrais me tourner. On présume, évidemment, qu'il s'agira de la famille nucléaire si intimement liée à cette période et à ce milieu. Mais à quel(s) membre(s) de cette famille s'adressent les promoteurs? Comment cette famille et ses membres sont-ils représentés dans les publicités?

Dans son texte sur l'histoire de la consommation, Magda Fahrni explique que «les consommateurs étudiés par les historiens sont souvent des consommatrices $^{80}$ ». En effet, un survol plus général des publicités publiées durant la période permet d'observer une importante présence des femmes comme auditoire auquel s'adressent et que mettent en vedette les publicitaires. Par exemple, Bell publie en 1955 une publicité au graphisme élaboré annonçant la connexion de Montréal à Laprairie. Une femme au téléphone y est représentée à l'avant-plan, tandis qu'un homme, à l'arrière-plan, se dirige vers sa voiture (figure 10) ${ }^{81}$. Comme le suggère cet exemple, et d'autres du même genre $^{82}$, les publicités sur l'habitation (l'occupation de la maison) - pour reprendre la terminologie de Fortin - visent et mettent généralement en scène des femmes. Est-ce à dire que les publicités relatives à l'habiter (donc à l'achat de la maison) viseront plutôt les hommes?

C'est certainement ce que suggère leur analyse. Du point de vue du genre, elles sont généralement assez neutres, mais les quelques références aux hommes ou aux femmes qu'on y retrouve pointent vers un discours qui s'adresse d'abord aux chefs de famille masculins. Ainsi, dans le très petit nombre de publicités où des femmes sont représentées, elles font office d'élément décoratif ${ }^{83}$ ou apparaissent comme la fidèle compagne de leur conjoint ${ }^{84}$. Un petit nombre d'autres publicités suggère clairement que les femmes joueront un rôle subordonné dans l'achat de la maison. Par exemple, dans une publicité publiée dans les deux langues et mettant en vedette le hockeyeur Maurice Richard, on explique que, lors d'un

80. M. Fahrni, «Explorer la consommation...», p. 468.

81. On y explique que c'est un développement qui « will bring friends, relatives and customers closer to you ", The Gazette, 11 juin 1955 , p. 2.

82. Voir, par exemple, cette publicité pour les automobiles De Soto qui explique que le modèle le plus récent est "for the man who's going places», alors que l'épouse et la fille du conducteur sont laissées derrière (The Gazette, 28 mai 1955, p. 11).

83. Voir par exemple La Patrie, 21 mai 1955, p. 53 et 17 septembre 1955, p. 50.

84. Voir par exemple cette publicité où un couple est représenté achetant une maison. Madame est en arrière-plan pendant que monsieur, en avant-plan, tend une liasse de billets à un vendeur que l'on ne voit pas. La Patrie, 27 août 1955, p. 45. Voir aussi La Patrie, $1^{\text {er }}$ octobre 1955, p. 47. Ce qui nous ramène d'ailleurs aux observations de Korinek sur les transformations des représentations des femmes dans les publicités durant cette période. V. Korinek, Roughing It in the Suburbs..., p. 159-161. 
Figure 10

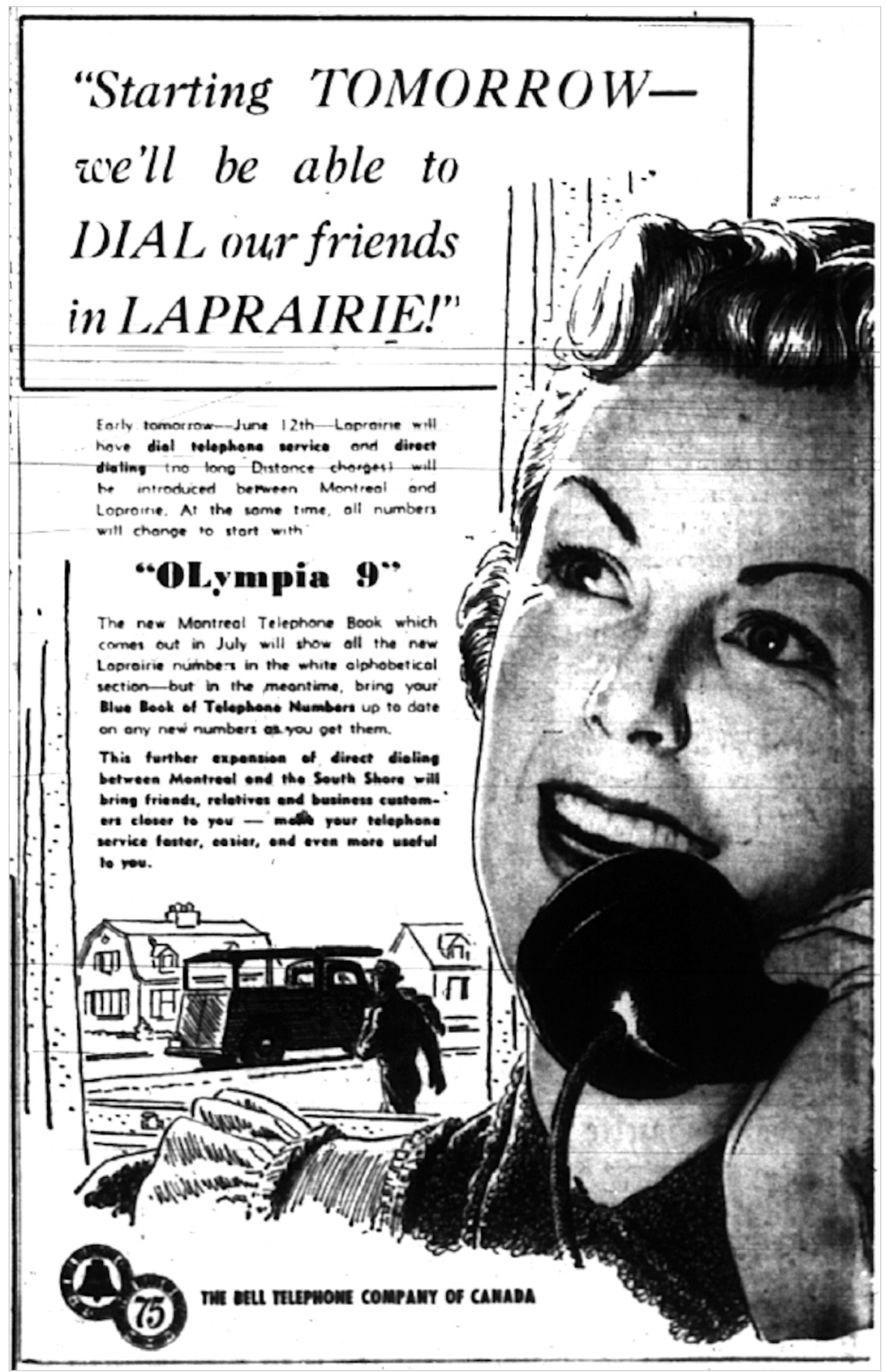

«Starting TOMORROW - we'll be able to DIAL our friends in LAPRAIRIE » : madame discute au téléphone dans la sphère domestique pendant que monsieur s'en va vaquer à ses affaires dans la sphère publique (The Gazette, II juin 1955, p. 2). 
événement au cours duquel seront présentées les trois maisons modèles du promoteur, une experte en économie domestique sera présente pour expliquer aux femmes les vertus des électroménagers au gaz pendant, on le présume, que l'homme du couple s'occupera des "vraies affaires " ${ }^{85}$. Un événement similaire a lieu à Fabreville en 1960 et, encore une fois, le promoteur explique qu'il y aura à «l'intention des dames démonstration d'arts ménagers et de décoration tous les après-midi et soir ${ }^{86}$ ". Ajoutons d'ailleurs, à cette occasion, que d'autres activités, dont des tours de poney, occuperont les enfants ${ }^{87}$.

Encore plus que les femmes, les enfants sont les grands absents des discours publicitaires étudiés. Les représentations d'enfants dans les publicités recensées se comptent sur les doigts d'une main ${ }^{88}$. Une publicité de $1965 \mathrm{du}$ promoteur Roger Pilon expliquera, par exemple, que l'achat d'une maison "will have an enormous influence on the happiness of your family and the harmonious development of your children ${ }^{89}$ ». On retrouvera un peu plus fréquemment, mais tout de même assez peu souvent, des références plus générales à la famille. Une publicité élaborée d’une page pour Duvernay Gardens décrira ses maisons comme «L'idéal pour la famille!» et fera une belle utilisation de rôles genrés pour les vendre (figure 11 : à consulter en ligne $)^{90}$. Ainsi, le père de famille, pipe en bouche, s'y exclame de joie devant leur faible prix et leur excellente construction, alors que la mère se réjouit de la modernité de leur cuisine et que quatre enfants annoncent: «Papa dit qu'on a tout fait pour que les rues soient sans danger!» C'est une approche que l'on retrouve dans une seule autre publicité de l'échantillon étudié, publiée en 1969 pour les Maisons Henri Dubord. Elle est également singulière, puisqu'elle est la seule à mettre en scène la figure émergente de l'adolescent, que l'on associe, de manière un peu inattendue, à un modèle de maison qui serait particulièrement bien adapté à ses goûts et que l'on affuble du nom improbable de "Le Discothèque »" .

Ce que cette dernière section suggère, c'est que les promoteurs et les publicitaires accordent une importance limitée à la famille - pourtant un élément théoriquement fondamental de l'idéal suburbain - lorsque vient

85. La Presse, 26 septembre 1959, p. 5 et The Gazette, 12 septembre 1959, p. 2.

86. La Presse, 17 septembre 1960 , p. 30.

87. Un événement similaire, axé sur la famille, a lieu l'année suivante à Châteauguay. The Gazette, 8 juillet 1961, p. 30.

88. Voir un rare exemple dans The Gazette, 16 janvier 1965, p. 39.

89. The Gazette, 2 octobre 1965 , p. 37.

90. La Presse, 6 juin 1959, p. 21.

91. The Gazette, 18 octobre 1969, p. 30. 
le moment de vendre des maisons aux consommateurs montréalais des années 1950-1960. On peut probablement lier ce désintéressement aux moyens limités de certains promoteurs, qui se contentent d'une publicité sobre du modèle type vu plus haut. Mais, même dans le cadre de campagnes plus ambitieuses et élaborées, ce sont les caractéristiques physiques de la maison et de son environnement ainsi que les modalités financières avantageuses et la profitabilité de l'investissement qui sont mises en relief. De fait, et même si c'est par défaut, on peut parler d'un discours qui s'adresse à un auditoire d'abord masculin, au chef de famille, mais sans se référer outre mesure à cette famille.

\section{DEUX SOLITUDES? VARIATIONS LINGUISTIQUES ET GÉOGRAPHIQUES}

Un dernier aspect de l'analyse mérite qu'on s'y attarde : l'importance du facteur linguistique. Force est de constater que, du point de vue du contenu des publicités du moins, il a un impact relativement limité et qui s'amoindrit au fil de la période. La principale différence qui saute aux yeux durant les années 1950 est probablement la présence, dans The Gazette, d'un grand nombre de publicités pour des appartements de luxe au centreville, publicités qui sont à peu près absentes des quotidiens francophones ${ }^{92}$. Ce qui est tout de même frappant, pour les années 1950, c'est le fait que même si les publicités étudiées se ressemblent, au niveau du contenu, les promoteurs et les développements observés dans la presse d'une des deux langues se retrouvent rarement de l'autre côté de la barrière linguistique. En d'autres mots, les petits entrepreneurs de l'époque s'adressent généralement uniquement à l'un des deux groupes linguistiques. C'est surtout à partir des années 1960 que l'on observera, de plus en plus fréquemment, des campagnes publicitaires reproduites dans les quotidiens anglophones et francophones de la métropole. Ainsi, toute une série d'annonces de plus en plus élaborées témoignent non seulement de l'ampleur grandissante des moyens dont disposent les prometteurs, mais aussi du fait que l'on assume que l'idéal suburbain n'a pas de langue ${ }^{93}$.

La question linguistique elle-même apparaît assez rarement dans le discours publicitaire. Un des rares cas relevés figure dans une publicité anglophone pour Laurier Park, à Candiac, qui mentionne que la commu-

92. Par exemple, une publicité de 1951 pour l'immeuble Croydon offre différents types d'appartement: «the Ambassador, the Statesman, the Midtowner, the Clubman». Notons la présence étonnante, pour l'époque, de la «business woman», qui est invitée à penser au «Midtowner». The Gazette, 3 mars 1951, p. 2.

93. Voir par exemple les publicités pour les maisons Mercury et Paramount que l'on retrouve dans La Presse, 20 mars 1965, p. 21, 30. Elles sont reprises à l'identique dans The Gazette de la même semaine. 
nauté a une «balanced English and French population ${ }^{94}$ ». Autrement, cette question est parfois évoquée de manière plus badine, comme dans cette publicité anglophone du promoteur Roger Pilon qui est surmontée du titre «TELLEMENT DIFFÉRENT! ! et qui explique que, dans son cas, "[the] accent is on "quality»"

Une publicité, reprise dans les deux langues, permet toutefois de mettre le doigt sur un des impacts majeurs de la question linguistique sur le déploiement de l'idéal suburbain dans la région montréalaise. Ainsi, une publicité du promoteur Parkview de janvier 1965 est reprise dans La Presse et The Gazette pour faire la promotion des sept développements sur lesquels travaille alors le promoteur (figures 12 et 13 : à consulter en ligne) ${ }^{96}$. À première vue, ces publicités sont identiques, mais on réalise rapidement que, dans chacune d'entre elles, la taille des encadrés varie d'une langue à l'autre: dans le quotidien anglophone, le développement de PointeClaire est mis en vedette, alors que celui de Brossard demeure en arrièreplan. Du côté de La Presse, c'est l'inverse. Ainsi, si les discours publicitaires analysés convergent nettement pour ce qui est de leur contenu, cet idéal suburbain commun semble être vécu selon une géographie qui, elle, diverge en partie selon la langue parlée.

Pour mieux visualiser ce phénomène, les publicités analysées ont été géolocalisées $^{97}$. Les cartes qui en résultent permettent de constater que les promoteurs, lorsqu'ils conçoivent leurs publicités, s'adaptent d'une certaine manière à la reproduction des deux solitudes dans la couronne suburbaine montréalaise et la nourrissent. Il faut toutefois relativiser ce phénomène. Ainsi, certains secteurs sont clairement associés, par les promoteurs, à un des deux principaux groupes linguistiques de la région. La partie est de la Rive-Sud de Montréal (Longueuil, Brossard) est essentiellement proposée aux acheteurs qui lisent la presse francophone, alors que l'ouest de l'île de Montréal (et tout particulièrement Pointe-Claire) est presque uniquement mis en marché pour un lectorat anglophone. Plusieurs secteurs témoignent toutefois d'une certaine mixité, du moins du point de vue publicitaire : c'est le cas de la Rive-Nord et de certains secteurs de l'île Jésus, mais aussi du secteur ouest de la Rive-Sud (de Candiac à Châteauguay) et de Pierrefonds et de Dollard-des-Ormeaux dans l'ouest

\footnotetext{
94. The Gazette, 17 avril 1965, p. 29.

95. The Gazette, 18 septembre 1965, p. 38.

96. The Gazette, 16 janvier 1965, p. 38 et La Presse, 16 janvier 1965, p. 31.

97. Les données et les cartes qui en résultent sont consultables, en accès libre, à cette adresse: $<$ http:// schema.uqam.ca/application/banlieues>
} 
de l'île. Bref, cette géolocalisation des données permet de constater assez clairement que les promoteurs et les publicitaires ont conscience des phénomènes d'agrégation linguistique qui se produisent dans le contexte de la suburbanisation de la région montréalaise et ils s'y adaptent de manière à mieux cibler leurs publicités. Ce travail est toutefois fait de manière nuancée, les promoteurs réalisant clairement que certains secteurs se prêtent mieux que d'autres à des campagnes qui s'adressent aux deux principales communautés linguistiques de la métropole.

\section{CONCLUSION}

La suburbanisation de la région montréalaise ne s'est pas subitement arrêtée en 1969. Elle se poursuit en ce moment même et ne semble pas vouloir s'essouffler (même si elle a considérablement changé de forme). Cela dit, mon analyse a permis d'explorer la façon dont elle est proposée aux Montréalais durant les premières phases de sa transformation en phénomène de masse au lendemain de la Seconde Guerre mondiale. J'ai ainsi mis en relief l'ampleur grandissante des projets résidentiels et des campagnes publicitaires qui en font la promotion. Cette expansion s'est notamment traduite par la diversification croissante de l'offre sur le plan socioéconomique: de maisons et de développements généralement modestes à des entreprises qui ont une offre pour toutes les bourses, qu'il s'agisse du «luxe à petit prix" ou de maisons qui viennent avec une carte de membre au country club local. De même, on a pu constater que les discours publicitaires, d'abord centrés sur la maison, se sont peu à peu élargis pour mettre en valeur l'environnement physique et social dans lequel s'inscrit cette résidence, et qu'ils s'adressent d'abord et avant tout à un acheteur identifié au père de famille, laissant à la marge sa conjointe et ses enfants. Enfin, la comparaison entre journaux francophones et anglophones a permis d'observer des discours convergents lorsque vient le moment de mettre en marché l'idéal suburbain à Montréal, même si c'est un idéal qui est visiblement vécu dans des géographies divergentes.

De manière plus globale, mon étude permet de saisir une facette spécifique de l'entrée de la société montréalaise dans la modernité nordaméricaine d'après-guerre, et de constater que si cette adhésion à l'idéal suburbain est indéniable, elle semble clairement plus tardive et plus modeste que dans d'autres régions du continent, quelque chose que lais- 
saient déjà clairement deviner, par exemple, les observations de PaulAndré Linteau sur l'impact de la frontière sur la façon dont le processus de suburbanisation est vécu au Canada et aux États-Unis ${ }^{98}$. Et cette différence est probablement amplifiée à Montréal par son statut de «ville de locataires".

Néanmoins, cet article a deux limites qui pointent vers de futures recherches. D'une part, j’y traite uniquement de la métropole montréalaise. Si le cas de la Vieille Capitale a déjà été abordé ailleurs ${ }^{99}$, il en va autrement des petites et moyennes villes de la province qui ont, elles aussi, vécu le processus de suburbanisation. Ces banlieues régionales ont peu ou pas été étudiées et mériteraient certainement qu'on s'y attarde, notamment pour observer l'impact du changement d'échelle sur leur développement. D’autre part, l'étude de ces publicités nous permet surtout de saisir la perspective des promoteurs immobiliers et des publicitaires, et d'analyser la lecture qu'ils font des désirs et aspirations du public qu'ils ciblent. Ces publicités nous en disent très peu sur ce que pensent réellement les acheteurs de ces résidences et sur la forme que prend leur expérience comme banlieusards de première génération. Derrière ces publicités se profile une réalité qui demeure relativement méconnue (et parfois caricaturée) par l'historiographie. Comme je l'ai observé ailleurs, les études récentes consacrées aux quartiers ouvriers québécois du $\mathrm{XX}^{\mathrm{e}}$ siècle ont une fâcheuse tendance à idéaliser, jusqu'à un certain point, la vie communautaire de ces espaces et à l'opposer au conformisme présumé des banlieues, qui contribueraient d'ailleurs à leur dévitalisation à partir des années $1950^{100}$. Pourtant, difficile à croire que l'expérience des milliers de banlieusards montréalais d'aprèsguerre ressemble au cauchemar suburbain que décrit Pierre Vallières dans Nègre blanc d'Amérique ${ }^{101}$. L'histoire orale permettrait, notamment, de mieux connaître l'expérience de ces pionniers de la suburbanisation d'après-guerre, de mieux saisir la diversité de leurs expériences et de mesurer la distance, très certainement assez grande, qui séparait la "promotion attrayante» des banlieues montréalaises de leur réalité concrète.

98. Paul-André Linteau, «Canadian Suburbanization in a North American Context. Does the Border Make a Difference?", Journal of Urban History, 13, 3 (1987), p. 252-274.

99. A. Fortin, C. Després et G. Vachon, dir., La banlieue revisitée...

100. Harold Bérubé, «La ville, quartier par quartier». Labour/Le Travail, 78 (automne 2016), p. 265-279. Les ouvrages dont il est question dans cette note critique sont Dale Gilbert, Vivre en quartier populaire. Saint-Sauveur, 1930-1980 (Québec, Septentrion, 2015); et Gilles Lauzon, Pointe-Saint-Charles. L'urbanisation d'un quartier ouvrier de Montréal, 1840-1930 (Québec, Septentrion, 2014).

101. Pierre Vallières, Nègre blanc d'Amérique (Montréal, Parti pris, 1968). 